\title{
Self-interacting dark matter in the solar system
}

\author{
Avijit K. Ganguly, ${ }^{1}$ Pankaj Jain, ${ }^{2}$ Subhayan Mandal, ${ }^{2}$ and Sarah Stokes ${ }^{3}$ \\ ${ }^{1}$ Haldia Institute of Technology, Haldia, India \\ ${ }^{2}$ Department of Physics, Indian Institute of Technology Kanpur 208016, INDIA \\ ${ }^{3}$ Department of Physics, California Institute of Technology Pasadena, California, USA \\ (Received 17 October 2006; revised manuscript received 8 May 2007; published 24 July 2007)
}

\begin{abstract}
Weakly coupled, almost massless, spin 0 particles have been predicted by many extensions of the standard model of particle physics. Recently, the PVLAS group observed a rotation of polarization of electromagnetic waves in vacuum in the presence of transverse magnetic field. This phenomenon is best explained by the existence of a weakly coupled light pseudoscalar particle. However, the coupling required by this experiment is much larger than the conventional astrophysical limits. Here we consider a hypothetical self-interacting pseudoscalar particle which couples weakly with visible matter. Assuming that these pseudoscalars pervade the galaxy, we show that the solar limits on the pseudoscalar-photon coupling can be evaded.
\end{abstract}

DOI: 10.1103/PhysRevD.76.025026

PACS numbers: $14.80 . \mathrm{Mz}, 92.60 . \mathrm{Vb}, 95.35 .+\mathrm{d}$

\section{INTRODUCTION}

In a recent paper Jain and Mandal [1] proposed a mechanism to evade the astrophysical bounds on light pseudoscalars. The authors argued that there are no experimental or astrophysical bounds on the pseudoscalar selfcouplings, which can, therefore, be treated as a free parameter. For sufficiently large, but perturbative, selfcoupling the mean free path of pseudoscalars can be very small inside the Sun, provided we have sufficiently high density of these particles. The authors argued that the pseudoscalars produced inside the Sun will accumulate due to self-couplings. The mechanism required the fragmentation of the pseudoscalars $\phi$ through the process $\phi\left(k_{1}\right)+\phi\left(k_{2}\right) \rightarrow \phi\left(k_{3}\right)+\phi\left(k_{4}\right)+\phi\left(k_{5}\right)+\phi\left(k_{6}\right)$. Some mechanism for energy loss as the particles propagate through the Sun was also required in order that the pseudoscalar particles are at sufficiently low temperature. Using this mechanism the authors argued the results of the PVLAS experiment [2] can be consistent with the astrophysical bounds [3] and with the results of the CAST experiment [4]. The PVLAS Collaboration finds a rotation of polarization of light in vacuum in the presence of a transverse magnetic field. If we interpret this rotation in terms of the coupling of a light pseudoscalar particle to photons we find that the allowed range of parameters are in conflict with the astrophysical bounds [3], although there is no conflict with any laboratory bounds [5]. The PVLAS result has motivated considerable theoretical work [6-15] as well as proposals for new experiments [16,17] and observations [18]. Furthermore new constraints have been imposed on axion monopole-dipole coupling [19] assuming the mass range observed in the PVLAS experiment.

Self-interacting dark matter has been considered by many authors. It was proposed in Ref. [20] due to its interesting consequences for cosmology. Constraints on such a dark matter candidate and its implications for cosmology have been further studied [21]. The dark matter studied in the present paper may not give a significant contribution to the energy density of the universe due to its very small mass. Significant contribution may arise only if the pseudoscalar field undergoes coherent oscillations around the minimum of the potential such as those expected for axion like fields [22]. This oscillatory field behaves just as nonrelativistic dark matter. Some evidence for self-interacting dark matter is also found in considering the galactic dark matter distribution [23]. The standard cold dark matter scenario leads to cuspy dark matter galactic halos, which are not in agreement with observations [24]. The self-interacting dark matter solves this problem and has been studied by many authors [25]. Alternatively in Ref. [26] it is claimed that the dark matter profile is in good agreement with cold dark matter predictions. It is interesting to determine whether the dark matter we consider can be the dominant component of the galactic dark matter. However, we postpone this question for future research and for now simply assume that the pseudoscalars we consider have densities negligible compared to the galactic dark matter density.

In the present paper we propose a simple mechanism for evading the astrophysical bounds. Our basic assumptions are as follows:

(1) The pseudoscalars have a perturbative, but relatively large, self-coupling.

(2) The pseudoscalars form some component of the galactic dark matter.

The second assumption implies that pseudoscalars at nearly zero temperature are present throughout the galaxy. This assumption is quite reasonable since pseudoscalars will be produced in the early universe and will be present today in the form of dark matter. Stars are formed in regions of dense molecular clouds where the average density of matter is much higher then the mean galactic density. It is very likely that the density of dark matter is also higher in these regions due to the large gravitational 
attraction. As the cloud of dust and gas collapses to form a star the pseudoscalar cloud will also collapse. This will lead to a high density of pseudoscalars inside the star. The mean free path of pseudoscalars produced inside the star may then be very small due to the their scattering on background pseudoscalars by the reaction $\phi\left(k_{1}\right)+$ $\phi\left(k_{2}\right) \rightarrow \phi\left(k_{3}\right)+\phi\left(k_{4}\right)$. Hence this mechanism can trap pseudoscalars inside the star and considerably limit the radiative transfer [27] that can occur through pseudoscalars. In the present paper we investigate this mechanism in order to determine whether it can indeed evade the astrophysical bounds. We will focus on the bounds imposed by considering the pseudoscalar production inside the core of the Sun. Our mechanism also applies to bounds due to red giants and supernovae. However, we postpone this discussion to future research.

\section{EVADING THE SOLAR BOUNDS}

The effective Lagrangian for the pseudoscalars can be written as

$$
\begin{aligned}
\mathcal{L}= & -\frac{1}{4} F_{\mu \nu} F^{\mu \nu}+\frac{1}{2} \partial_{\mu} \phi \partial^{\mu} \phi-\frac{1}{2} m^{2} \phi^{2} \\
& +\frac{1}{4 M_{\phi}} \phi F_{\mu \nu} \tilde{F}^{\mu \nu}-\frac{\lambda}{4 !} \phi^{4},
\end{aligned}
$$

where we have assumed a self-coupling term besides their interaction with photons. Assuming that the results of PVLAS experiment can be explained in terms of pseudoscalar-photon mixing, the parameter $M_{\phi}$ and the pseudoscalar mass $m_{\phi}$, respectively, lie in the range $1 \times$ $10^{5} \mathrm{GeV} \leq M_{\phi} \leq 6 \times 10^{5} \mathrm{GeV}$ and $0.7 \mathrm{meV} \leq m_{\phi} \leq$ $2 \mathrm{meV}$ [2]. We assume that the self-coupling $\alpha_{\lambda}=$ $\lambda^{2} / 4 \pi \leq 0.1$, so that it can be treated perturbatively. The quadratically divergent loop corrections in scalar field theories lead to well-known fine tuning problems. The $\phi^{4}$ coupling, for example, leads to a quadratically divergent contribution to the mass of the pseudoscalar particle at one loop order. However, in the present case we should consider this Lagrangian as an effective phenomenological model and hence we ignore these complications.

The density of dark matter in the galaxy may be parametrized as [28]

$$
\rho(r)=\frac{C_{o}}{a^{2}+r^{2}} .
$$

We may assume that the pseudoscalars form a fraction $\xi$ of the galactic dark matter, where $C_{o}=4.6 \times 10^{8} M_{\odot} \mathrm{kpc}^{-1}$ and $a=2.8 \mathrm{kpc}$. Using the mass of the pseudoscalar to be about $10^{-3} \mathrm{eV}$ and the distance of earth from the galactic center $r=8 \mathrm{kpc}$, we estimate the number density $n=$ $2.4 \xi 10^{11} \mathrm{~cm}^{-3}$. We assume $\xi$ to be less than 0.1 so that pseudoscalars contribute negligibly to the galactic dark matter density. This parametrization of the density profile is sufficiently reliable for our purpose. It correctly models the expected $1 / r^{2}$ behavior at large distances and becomes constant at small distances. The simulated dark matter distributions for several different models such as the standard cold dark matter and cold dark matter with self interaction are shown in Ref. [23]. The resulting density obtained for large $r$ is in good agreement with that obtained with the parameterization given in Eq. (2) for these models. Hence we find that a wide range of reasonable models of galactic dark matter produce a density in good agreement with what is obtained from Eq. (2) at the position of the Earth inside the Milky Way.

The pseudoscalar density may be higher at the site of star formation due to the higher concentration of matter in this region. We set the pseudoscalar number density in a region which leads to the formation of the Sun to be a factor $\eta$ times its galactic density. This region, which contained the primordial cloud of gas and dust whose collapse leads to the formation of the solar system, is expected to be of size larger than 100-1000 AU. As the star forms the pseudoscalars in this region may also collapse onto the star. The precise pseudoscalar density profile will be determined later by requiring that the solar system, including the pseudoscalar gas, are in steady state. Here we make an order of magnitude estimate of the pseudoscalar number density inside the Sun, assuming that almost all the pseudoscalars within a radius $R_{S} \approx 1000 \mathrm{AU}$ collapse onto the Sun. The resulting number density of pseudoscalars inside the Sun is found to be

$$
n_{\phi \odot} \approx 2.4 \xi \eta 10^{26}\left[\frac{R_{S}}{1000 \mathrm{AU}}\right]^{3} \mathrm{~cm}^{-3} .
$$

As the star collapses the temperature in the core increases, eventually leading to nuclear reactions. The photons in the core then start producing pseudoscalars through the Primakoff process [1]. The temperature of the pseudoscalars inside the Sun also increases, partially due to the collapse of pseudoscalars and partially due to production of high energy pseudoscalars by the Primakoff process. We can compute the kinetic energy of pseudoscalars as they reach the solar radius from nearly infinite distance. Assuming that their initial kinetic energy is almost zero we find that the final kinetic energy is $3.4 \times 10^{-21} \mathrm{ergs}$ which is equivalent to a temperature of $2.5 \times 10^{-5} \mathrm{~K}$. Hence this predicts a relatively cool pseudoscalar gas. This result may be significantly modified due to conversion of photons into pseudoscalars inside the Sun. As already mentioned, the final density profile is obtained later assuming steady state of the solar system and does not rely on these rough estimates.

The typical energy of a pseudoscalar produced inside the core of the Sun, assuming present day conditions, is of order $1 \mathrm{KeV}$. This is much larger than the kinetic energy acquired by pseudoscalars due to conversion of potential energy. We expect that the pseudoscalars will eventually reach a steady state, where the outward pressure balances 
the gravitational attraction. Furthermore, there should be no net exchange of energy between the photon and the pseudoscalar gas. The rate at which photons loose energy by conversion to pseudoscalars is given by

$$
\dot{\epsilon}=\frac{n_{e} n_{\gamma}}{\rho}\left\langle c \int E_{\phi} \frac{d \sigma}{d E_{\phi}} d E_{\phi}\right\rangle
$$

where $\rho$ is the density of the medium and the angular brackets denote thermal averaging. Equating this to the rate at which photons are gaining energy due to the conversion of pseudoscalars into photons we find

$$
\frac{n_{e} n_{\gamma}}{\rho}\left\langle c \int E_{\phi} \frac{d \sigma}{d E_{\phi}} d E_{\phi}\right\rangle=\frac{n_{e} n_{\phi}}{\rho}\left\langle v \int E_{\gamma} \frac{d \sigma}{d E_{\gamma}} d E_{\gamma}\right\rangle \text {. }
$$

It is clear that the maximum temperature that the pseudoscalars can acquire is equal to the photon temperature. The pseudoscalars would acquire this temperature if in thermal equilibrium with the photon gas. At the temperatures corresponding to the solar interior the pseudoscalars would be relativistic and their number density would be equal to the photon number density. This argument gives us a number density in the core of the Sun to be of the order of $10^{23} \mathrm{~cm}^{-3}$. This is really a lower bound since, as discussed later, the temperature of pseudoscalars may be lower. In this case their number density has to be higher in order to maintain a steady state with the photon gas.

The pseudoscalars may not be in thermal equilibrium inside the Sun. This is due to the fragmentation process

$$
\phi\left(k_{1}\right)+\phi\left(k_{2}\right) \rightarrow \phi\left(k_{3}\right)+\phi\left(k_{4}\right)+\phi\left(k_{5}\right)+\phi\left(k_{6}\right),
$$

which occurs through the Feynman diagram shown in Fig. 1. If we assume coupling $\lambda$ of order unity, the cross section for this process at center of mass energy $E, \sigma \sim$ $\alpha_{\lambda}^{2} / E^{2}$, is much larger than the cross section for the inverse Primakoff process for the conversion of pseudoscalars to photons. Hence pseudoscalars would fragment rather than convert to photons. The fragmentation process will stop only when the pseudoscalar energy becomes comparable to their mass. Hence we expect the pseudoscalars inside the Sun will have energies comparable to their mass and will not be ultrarelativistic.

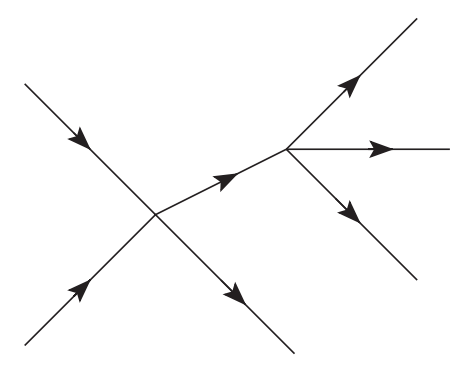

FIG. 1. Feynman diagram for the fragmentation process $\phi \phi \rightarrow \phi \phi \phi \phi$.
The cross section $\sigma$ for the process $\phi\left(k_{1}\right)+\phi\left(k_{2}\right) \rightarrow$ $\phi\left(k_{3}\right)+\phi\left(k_{4}\right)$ at leading order in perturbation theory is given by [1]

$$
\sigma_{\phi \phi}=\frac{\alpha_{\lambda}}{8 E_{c m}^{2}} \approx 1.2 \times 10^{11} \alpha_{\lambda}\left[\frac{10^{-6} \mathrm{GeV}}{E_{c m}}\right]^{2} \mathrm{GeV}^{-2} .
$$

Assuming a center of mass energy of $1 \mathrm{KeV}$, we find the mean free path $l \approx 2 \times 10^{-7} / \alpha_{\lambda} \mathrm{cm}$. With $\alpha_{\lambda}=0.1$, this gives a mean free path $l \approx 2 \times 10^{-6} \mathrm{~cm}$, which is much smaller than the mean free path of photons inside the core. Hence we expect that pseudoscalars will contribute negligibly to radiative transport in comparison to photons and this mechanism evades the limits imposed by considering the cooling rate of the Sun.

As seen above, the pseudoscalars are essentially trapped inside the Sun and unable to escape freely. Because of the fragmentation process they may not be in equilibrium with the rest of the system inside the Sun. However, in steady state there would be no exchange of energy between pseudoscalars and the remaining particles. Because of the fragmentation process a pseudoscalar of energy $E$ will produce roughly $E / m_{\phi}$ pseudoscalars. The typical energy of a pseudoscalar produced in the outer regions of the Sun by conversion from photons is of order $1 \mathrm{eV}$. The temperature of the pseudoscalar gas is of order of their mass, i.e. of order $0.001 \mathrm{eV}$. Hence a photon produced by pseudoscalars will have energy of order $0.001 \mathrm{eV}$. Since the cross sections for Primakoff and the inverse Primakoff process are roughly equal, we expect that, in steady state, the number density of pseudoscalars in the outer regions of the Sun is of order 1000 times the photon density. The pseudoscalars will, therefore, form a relatively cold and dense gas. For a certain range of self-coupling it cannot be described as an ideal gas since its range of interaction may be much larger than the typical separation between particles. The pseudoscalar gas will maintain steady state with the remaining particles by producing large number of low energy photons which will be quickly absorbed by the fermions inside the Sun and hence will equilibrate.

Outside the Sun the photons undergo free streaming. The pseudoscalars, however, are unable to propagate freely due to the background distribution of pseudoscalars. We next get an estimate of the pseudoscalar density profile outside the Sun by considering two limiting cases. We first assume that pseudoscalars are in exact thermal equilibrium with the remaining particles inside the Sun. In the other extreme we assume that the fragmentation process dominates.

\section{A. Thermal equilibrium}

Here we assume that pseudoscalars are in thermal equilibrium inside the Sun. This provides an important limiting case, although for the range of couplings that we are studying this may never be realized. In this limit the pseudosca- 
lars are ultrarelativistic near the surface of the Sun. The number density of pseudoscalars is obtained by Eq. (5) and is found to be the same as that of photons throughout the radius of the Sun. We can compute the mean free path of pseudoscalars throughout the solar radius using this density profile. It is found to be of order $10^{-7} / \alpha_{\lambda}$ c.m. near the core and increases to about $10^{-4} / \alpha_{\lambda}$ near the solar surface. Hence for a wide range of values of $\alpha_{\lambda}$ it is much smaller than the photon mean free path and the energy transport will occur primarily by photon emission. Let $R_{\odot}$ denote the solar radius. For $r>R_{\odot}$, we assume, for simplicity, that we can describe pseudoscalars as an ideal Bose gas. At $r=R_{\odot}$ we impose the boundary condition that the pseudoscalar temperature is equal to the temperature at the surface of the Sun. The equation for the pressure gradient can be written as [29]

$$
\frac{d P}{d r}=-\frac{G M(r)}{r^{2}}\left(\rho_{\phi}(r)+P(r)\right),
$$

where $\rho_{\phi}$ is the energy density of the pseudoscalars, $P$ the pressure and

$$
M(r)=4 \pi \int_{0}^{r} d r^{\prime} r^{\prime 2} \rho_{\phi}\left(r^{\prime}\right) .
$$

We point out that we are interested in the solution for $r>$ $R_{\odot}$, where we neglect all other particles except the pseudoscalars. In Eq. (7) we have kept the dominant relativistic corrections. For $r>R_{\odot}$, we have $P(r)=\rho_{\phi}(r) / 3$ and we may assume that $M(r)=M_{\odot}$. The solution can be written as

$$
\rho_{\phi}(r)=\rho_{\phi}\left(R_{\odot}\right) \exp \left[-4 G M_{\odot}\left(\frac{1}{R_{\odot}}-\frac{1}{r}\right)\right] .
$$

This essentially means that $\rho_{\phi}(r)$ remains roughly constant inside the solar system. The solution is not valid as we go to distances beyond the solar system since then we need to take into account gravitational effects of other objects in the Milky Way. The pseudoscalar density profile obtained for $r>R_{\odot}$ again implies a very small mean free path in this region. Hence the pseudoscalars are unable to propagate freely throughout the entire solar system and the standard solar model remains unaffected by the presence of these particles.

\section{B. Fragmentation}

We next consider the other extreme where the fragmentation dominates. In this case we assume that the energy per particle of the pseudoscalars is of the order of their mass due to the fragmentation process. The pseudoscalars may, therefore, not be ultrarelativistic. In order to get an estimate of the density profile for $r>R_{\odot}$ we assume that they are nonrelativisitic in the outer regions of the Sun, such that their temperature is smaller than their mass. The temperature of the photon gas near the surface is about $10^{4} \mathrm{~K}$. Since the mass of the pseudoscalar is about
$10^{-3} \mathrm{eV}$, we expect that the pseudoscalar temperature $T_{\phi}<10 \mathrm{~K}$. By using Eq. (5) and the fact that the cross sections for the direct and the inverse Primakoff process are roughly equal, we find that the number density of pseudoscalars at the surface $n_{\phi}\left(R_{\odot}\right)>10^{3} n_{\gamma}$. The factor $10^{3}$ arises due to the ratio $T_{\gamma} / T_{\phi}$. We may similarly obtain an estimate of the density profile inside the entire radius of the Sun. The precise value is not relevant for our purpose. The number density is certainly at least as large as the photon number density throughout the Sun and that is enough to suppress the energy loss through pseudoscalar emission to negligible values.

The equation for hydrostatic equilibrium in this case is given by

$$
\frac{d P}{d r}=-\frac{G M(r) \rho_{\phi}(r)}{r^{2}} .
$$

We next need to specify the nature of change that the medium undergoes. We shall be interested in the pseudoscalar gas for $r>R_{\odot}$. In this region we assume that it is governed entirely by its self-coupling and its interaction with other particles is negligible. Because of the large cross section of the process $\phi \phi \rightarrow \phi \phi$ it is clear that radiative transport of energy is negligible. This is because pseudoscalars are not able to propagate freely in this medium. The medium is therefore likely to undergo adiabatic changes and hence will reach a steady state such that $P \rho_{\phi}^{-\gamma}=$ const, where $\gamma \equiv c_{P} / c_{V}$. For generality we assume a polytropic equation of state

$$
P \rho_{\phi}^{-\gamma^{\prime}}=\mathcal{K},
$$

which models a large number of processes for different values of the index $\gamma^{\prime}$. For adiabatic change $\gamma^{\prime}=\gamma$. We make the change of variables $\rho_{\phi}(r)^{\left(\gamma^{\prime}-1\right)}=z(r)$ and $x=$ $r / \sqrt{\alpha}$ where $\alpha=\mathcal{K} \gamma^{\prime} /\left[4 \pi\left(\gamma^{\prime}-1\right) G\right]$. In terms of $x$ and $z$ the equation for hydrostatic equilibrium can be brought into the form of Lane-Emden equation,

$$
\frac{1}{x^{2}} \frac{d}{d x}\left(x^{2} \frac{d z(x)}{d x}\right)+[z(x)]^{1 /\left(\gamma^{\prime}-1\right)}=0 .
$$

In order to bring the equation into dimensionless form it is convenient to use the scaled variables $\bar{z}$ and $\lambda$, defined by

$$
z(x)=\bar{z}(x) \rho_{\phi}\left(R_{\odot}\right)^{(\gamma-1)}, \quad x=\lambda \rho_{\phi}\left(R_{\odot}\right)^{(\gamma-2) / 2},
$$

where $\rho_{\phi}\left(R_{\odot}\right)$ is the pseudoscalar density at the surface of the Sun. The equation in terms of $\bar{z}$ and $\lambda$ is the same as Eq. (12) with $x$ and $z$ replaced by $\lambda$ and $\bar{z}$, respectively. The boundary conditions to be imposed are

$$
\bar{z}\left(\lambda_{o}\right)=1, \quad \frac{d \bar{z}}{d \lambda}\left(\lambda_{o}\right)=\delta,
$$

where $\lambda_{o}$ is the value of the dimensionless variable $\lambda$ at the surface of the Sun $r=R_{\odot}$ and $\delta$ can be determined by relating $d \bar{z} / d \lambda$ to $d \rho_{\phi} / d r$ at the surface of the Sun. For the 


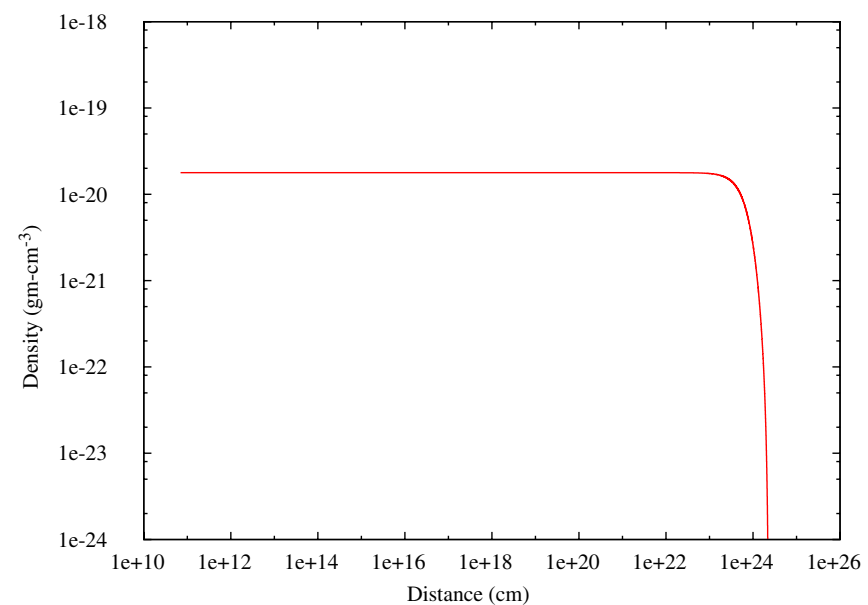

FIG. 2 (color online). Density profile of pseudoscalars with distance.

values given above, we find $\delta \approx 1 \times 10^{-40}$. The resulting density profile is plotted in Fig. 2. In obtaining this result we have set $\gamma^{\prime}=\gamma=5 / 3$. In the present case the medium is essentially opaque to the propagation of pseudoscalars due to the large scattering cross section of the process $\phi \phi \rightarrow \phi \phi$. Hence we can safely assume negligible energy transport and use the adiabatic equation of state.

We find that the density distribution is constant from the edge of the Sun up to a distance of order $100 \mathrm{Kpc}$. Of course our solution is no longer valid once we are outside the solar system since we have neglected the presence of other objects in the Milky Way. Beyond a certain distance the pseudoscalar density has to match the galactic pseudoscalar density. However, we do not address the issue of galactic density profile in this paper. We point out that even at very large distances from the Sun, where the pseudoscalar density reaches its galactic values, the mean free path of pseudoscalars is still very small, of order of a c.m., for a suitable choice of parameters $\alpha_{\lambda}$ and $\xi$. Hence even here pseudoscalars may not be able to propagate freely for a wide range of these parameters.

It is easy to see that the density has to remain constant for a large range of values of $r$ above the surface of the Sun. For this purpose we start with the basic equation for pressure gradient, Eq. (10). Using the polytropic equation of state, Eq. (11), we convert this into a differential equation for $\rho_{\phi}$

$$
\frac{d \rho_{\phi}}{d r}=-\frac{G M(r)}{r^{2} \mathcal{K} \gamma}\left(\rho_{\phi}(r)\right)^{2-\gamma} .
$$

The total mass contained within a distance $r, M(r)$, may be split up as

$$
M(r)=M_{\odot}+\int_{R_{\odot}}^{r} 4 \pi r^{\prime 2} d r^{\prime} \rho_{\phi}\left(r^{\prime}\right) .
$$

The second term on the right-hand side is negligible compared to the first term as long as $r$ is comparable to or only a few orders of magnitude larger than $R_{\odot}$. We may, therefore, drop the second term for a wide range of values of $r$. With this approximation the equation can be integrated analytically and we find

$$
\rho_{\phi}(r)^{\gamma-1}-\rho_{\phi}\left(R_{\odot}\right)^{\gamma-1}=-(\gamma-1) \frac{G M_{\odot}}{\mathcal{K} \gamma}\left(\frac{1}{R_{\odot}}-\frac{1}{r}\right) .
$$

We find that the right-hand side is negligible for $r>R_{\odot}$ and hence for a wide range of values of $r$ the solution is $\rho_{\phi}(r) \approx \rho_{\phi}\left(R_{\odot}\right)$.

The number densities obtained for all values of $r$ are again sufficiently large so that the mean free path of pseudoscalars is much smaller than that of photons. Hence the energy loss is expected to arise primarily through photon emission and the standard solar model will remain unaffected.

It is interesting that the form of the solution for a wide range of values of $r$ is the same as that obtained in Sec. II A assuming thermal equilibrium throughout the Sun. The number density is essentially constant within the entire solar system for $r>R_{\odot}$. The precise number is obtained by the boundary condition imposed at the surface of the Sun. Furthermore, our mechanism for evading astrophysical bounds on pseudoscalar coupling and mass values is found to be applicable independent of the assumptions used to obtain the density profile inside the Sun.

We next check if our solution is consistent with the constraints imposed by the Pioneer anomaly [30]. We find that the solution obtained is not in conflict with the maximum allowed dark matter density inside the solar system [31,32]. However, the density is about an order of magnitude smaller compared to what is required to reproduce the anomalous acceleration of pioneer [30] crafts 10 and 11, towards the Sun. We may achieve higher densities if the pseudoscalars have a temperature of the order of $1 / 2 \mathrm{~K}$ at the surface of the Sun, instead of $10 \mathrm{~K}$, which we assumed. This can arise if the pseudoscalars also lose energy as they propagate inside the Sun [1]. By using Eq. (5) we find that this gives a higher pseudoscalar density at the surface of the Sun by a factor of about 20 . This leads to an increase in the density in such a way that it exactly matches the pioneer anomaly data.

\section{MEDIUM MODIFICATIONS}

We next address an important consistency check of our proposal. It is important to determine how the presence of background pseudoscalars affect the results of the PVLAS experiment. The density of pseudoscalars is relatively small and due to their weak coupling with photons, they will not significantly affect the propagation of photons. The dominant effect is modification of the pseudoscalar self energy. The medium modifications can be taken into account by using the modified mass $m_{T}^{2}=m_{\phi}^{2}+\delta m_{\mathrm{th}}^{2}$, 
where the thermal mass [33]

$$
\delta \mathrm{m}_{\mathrm{th}}^{2}=\frac{\lambda}{4 \pi^{2}} \int_{0}^{\infty} \mathrm{k}^{2} \mathrm{dk} \frac{1}{\mathrm{E}_{\mathrm{k}}} \frac{1}{\mathrm{e}^{\beta \mathrm{E}_{\mathrm{k}}}-1},
$$

where $E_{k}=\sqrt{k^{2}+m_{\phi}^{2}}$ and $\beta=1 / T$. In the high temperature limit, i.e., $T \gg m_{0}$, the result is straightforward and it turns out to be

$$
\delta \mathrm{m}_{\mathrm{th}}^{2}=\frac{\lambda \mathrm{T}^{2}}{24} .
$$

In the low temperature limit $\beta \rightarrow \infty$, we find

$$
\delta \mathrm{m}_{\mathrm{th}}^{2} \sim \frac{\lambda}{4 \pi^{2}} \sqrt{\frac{\pi \mathrm{mT}}{2}} \mathrm{Te}^{-\mathrm{m} / \mathrm{T}} .
$$

At the position of Earth, we expect that the temperature would be at most as high as the mass of the pseudoscalar particle, due to the fragmentation process. Hence the change in the pseudoscalar mass due to thermal effects would be small as long as $\lambda<1$. The modification in mass could be large if for some reason the fragmentation process is not very effective. In this case we should interpret the mass extracted by the PVLAS experiment as being domi- nantly the thermal mass. In either case, our mechanism for evading astrophysical bounds remains applicable.

\section{SUMMARY AND CONCLUSIONS}

We have shown that the solar astrophysical limits can be evaded provided we assume a sufficiently large value of the self-coupling of pseudoscalars and that pseudoscalars form some component of the galactic dark matter. The selfcoupling required is within the perturbative regime and the galactic density of pseudoscalars is assumed to be a negligible fraction of the dark matter density. Inside the Sun the maximum possible value of pseudoscalar temperature at any radius $r$ is equal to the solar temperature at that point. The corresponding mean free path of pseudoscalars is found to be sufficiently small so that they contribute negligibly to radiative transfer. We have shown that outside the Sun the density profile of pseudoscalars is roughly uniform. The density outside the star is found to be such that the mean free path of pseudoscalars is smaller than a c.m. for a wide range of values of the self-coupling $\alpha_{\lambda}$. Hence the pseudoscalars cannot escape freely from the Sun and the standard solar model remains unaffected. The solar limits on the pseudoscalar-photon coupling are, therefore, evaded.
[1] P. Jain and S. Mandal, Int. J. Mod. Phys. D 15, 2095 (2006).

[2] E. Zavattini et al. (PVLAS Collaboration), Phys. Rev. Lett. 96, 110406 (2006).

[3] D. Dicus, E. Kolb, V. Teplitz, and R. Wagoner, Phys. Rev. D 18, 1829 (1978); 22, 839 (1980); G. Raffelt and D. Dearborn, Phys. Rev. D 36, 2211 (1987); D. Dearborn, D. Schramm, and G. Steigman, Phys. Rev. Lett. 56, 26 (1986); J. Ellis and K. Olive, Phys. Lett. B 193, 525 (1987); G. Raffelt and D. Seckel, Phys. Rev. Lett. 60, 1793 (1988); M. Turner, Phys. Rev. Lett. 60, 1797 (1988); H.-T. Janka et al., Phys. Rev. Lett. 76, 2621 (1996); W. Keil et al., Phys. Rev. D 56, 2419 (1997); M. I. Vysotsky, Ya. B. Zeldovich, M. Yu. Khlopov, and V. M. Chechetkin, JETP Lett. 27, 502 (1978).

[4] K. Zioutas et al. (CAST Collaboration), Phys. Rev. Lett. 94, 121301 (2005).

[5] R. Cameron et al., Phys. Rev. D 47, 3707 (1993).

[6] A. Ringwald, J. Phys.: Conf. Ser. 39, 197 (2006); A. Dupays, C. Rizzo, M. Roncadelli, and G. Bignami, Phys. Rev. Lett. 95, 211302 (2005).

[7] E. Masso and J. Redondo, J. Cosmol. Astropart. Phys. 09 (2005) 015; Phys. Rev. Lett. 97, 151802 (2006); Contribution to the Rencontres de Physique de la Vallee d'Aoste, La Thuile, Aosta Valley, 2006, arXiv:hep-ph/ 0606164; AIP Conf. Proc. 878, 387 (2006).

[8] E. Masso, arXiv:hepph/ 0607215.
[9] I. Antoniadis, A. Boyarsky, and O. Ruchayskiy, arXiv:hep$\mathrm{ph} / 0606306$.

[10] N. Chatillon and A. Pinzul, Nucl. Phys. B764, 95 (2007).

[11] R. N. Mohapatra and S. Nasri, Phys. Rev. Lett. 98, 050402 (2007).

[12] H. Gies, J. Jaeckel, and A. Ringwald, Phys. Rev. Lett. 97, 140402 (2006).

[13] E. Gabrielli, K. Huitu, and S. Roy, Phys. Rev. D 74, 073002 (2006).

[14] B. A. Dobrescu and I. Mocioiu, J. High Energy Phys. 11 (2006) 005.

[15] J. Redondo, Contribution to the QCD06 International Conference, Montpellier, July 2006, arXiv:hep-ph/ 0610213.

[16] J. Jaeckel, E. Masso, J. Redondo, A. Ringwald, and F. Takahashi, Contribution to the Proceedings of the Rencontres des Moriond: Contents and Structures of the Universe, arXiv:hepph/ 0605313; Phys. Rev. D 75, 013004 (2007); A. K. Ganguly, Ann. Phys. (N.Y.) 321, 1457 (2006).

[17] U. Koetz, A. Ringwald, and T. Tschentscher, arXiv:hepex/0606058.

[18] M. Fairbairn, T. Rashba, and S. Troitsky, Phys. Rev. Lett. 98, 201801 (2007).

[19] S. Baessler et al., Phys. Rev. D 75, 075006 (2007).

[20] E. D. Carlson, M. E. Machacek, and L. J. Hall, Astrophys. J. 452, 495 (1995). 
[21] A. A. de Laix, R. J. Scherrer, and R. K. Schaefer, Astrophys. J. 452, 495 (1995); M.E. Machacek, Astrophys. J. 431, 41 (1994).

[22] J. Preskill, M. B. Wise, and F. Wilczek, Phys. Lett. 120B, 127 (1983); L. Abbott and P. Sikivie, Phys. Lett. 120B, 133 (1983); M. Dine and W. Fischler, Phys. Lett. 120B, 137 (1983); P. J. Steinhardt and M. S. Turner, Phys. Lett. 129B, 51 (1983); M. S. Turner, Phys. Rev. D 32, 843 (1985).

[23] D. N. Spergel and P. J. Steinhardt, Phys. Rev. Lett. 84, 3760 (2000); R. Dave, D. N. Spergel, P. J. Steinhardt, and B. D. Wandelt, Astrophys. J. 547, 574 (2001).

[24] R. A. Swaters, B. F. Madore, and M. Trewhella, Astrophys. J. 531, L107 (2000).

[25] S. Hannestad and R. J. Scherrer, Phys. Rev. D 62, 043522 (2000); M. C. Bento, O. Bertolami, R. Rosenfeld, and L. Teodoro, Phys. Rev. D 62, 041302 (2000); S. Balberg, S. L. Shapiro, and S. Inagaki, Astrophys. J. 568, 475 (2002); C. Firmani, E. D’Onghia, V. Avila-Reese, G.
Chincarini, and X. Hernandez, Mon. Not. R. Astron. Soc. 315, L29 (2000); E. D'Onghia and A. Burkert, Astrophys. J. 586, 12 (2003); S. Mitra, Phys. Rev. D 71, 121302 (2005); Kyung-Jin Ahn and P. R. Shapiro, Mon. Not. R. Astron. Soc. 363, 1092 (2005).

[26] R. Jimenez, L. Verde, and S. P. Oh, Mon. Not. R. Astron. Soc. 339, 243 (2003).

[27] G. G. Raffelt and G. D. Starkman, Phys. Rev. D 40, 942 (1989).

[28] B. W. Carrol and D. A. Ostlie, An Introduction to Modern Astrophysics (Addison-Wesley, Reading, MA, 1996).

[29] S. Weinberg, Gravitation and Cosmology (Wiley \& Sons, New York, 1972).

[30] J. D. Anderson et al., Phys. Rev. D 65, 082004 (2002).

[31] S. G. Turyshev et al., Int. J. Mod. Phys. D 15, 1 (2006).

[32] R. Foot and R. R. Volkas, Phys. Lett. B 517, 13 (2001).

[33] A. Das, Finite Temperature Field Theory (World Scientific, Singapore, 1997). 